\title{
Comparative study of two techniques on some nonlinear problems based ussing conformable derivative
}

https://doi.org/10.1515/nleng-2020-0030

Received Feb 24, 2020; accepted Nov 19, 2020.

Abstract: In this paper, three eminent types of timefractional nonlinear partial differential equations are considered, which are the fractional foam drainage equation, fractional Gardner equation, and fractional FornbergWhitham equation in the sense of conformable fractional derivative. The approximate solutions of these considered problems are constructed and discussed using the conformable fractional variational iteration method and conformable fractional reduced differential transform method. The conformable derivative is one of the admirable choices to handle nonlinear physical problems of different fields of interest. Comparisons of approximate solution obtained by two techniques, to each other and with the exact solutions are also presented and affirm that the considered methods are efficient and reliable techniques to study other nonlinear fractional equations and models in the sense of conformable derivative. To explain the effects of several parameters and variables on the movement, the approximate results are shown in tables and two-and three-dimensional surface graphs.

Keywords: fractional calculus, conformable fractional derivative, reduced differential transform method, variational iteration method

\section{Introduction}

Fractional partial differential equations are used to model most of the natural problems, such as finance, physical, chemical, fluid dynamics, biological and other fields of engineering. For diagnosing these physical models as well as in addition follow these physical models in realistic med-

\footnotetext{
Aniqa Zulfiqar, Department of Mathematics, Faculty of Science, University of Gujrat, Pakistan

*Corresponding Author: Jamshad Ahmad, Department of Mathematics, Faculty of Science, University of Gujrat, Pakistan, E-mail: jamshadahmadm@gmail.com
}

ical studies, it is essential to discover their approximate and exact solutions. In calculus a lot of numerical, analytic and approximate methods are developed for nonlinear models [1-7].

There are miscellaneous researches correlated to this derivative. Atangana et al. [8] give a few definitions of the conformable derivative and time-space fractional heat differential equations being resolved with the aid of the conformable derivative delivered by Cenesiz and Kurt [9]. New exact solutions of conformable Burgers' type equations obtained by Çenesiz et al. [10]. Conformable differential method was introduced by Ünal and Gökdoğan [11]. In addition, the conformable fractional method has been carried out to many models with the aid of many authors [1215]. By using conformable operator Yavuz explains the dynamical behaviors of models $[16,17]$. The conformable fractional derivative is taken into consideration for this examine because it is simple for the calculation. A Chinese professor He $[18,19]$ introduced variational iteration method. Such as linear and nonlinear wave equation, systems of fourth-order differential equations are solved by the variational iteration method [20]. Conformable variational iteration method is built on the conformable derivative for fractional ordinary differential equations and fractional partial differential equations [21].

Keskin and Oturanc [22, 23] introduced reduced differential transform method. The technique gives a dependable and proficient process for extensive kind of chemical engineering, medical and fluid model problems, inclusive of fractional order or non-fractional partial differential equations [24]. By the definition of conformable derivative, Acan et al. [25] offered a new form of reduced differential transform technique that is known as conformable reduced differential transform, implemented to a few fractional differential equations.

Take a look, foam drainage equation is very momentous because many technological and commercial programs were developed, which consist cleansing, water purification, mineral extraction. Numerical solution of fractional differential equations is supplied in [26]. Traveling Wave solution of fractional differential equations with the 
aid of using rational $\left(\mathrm{G}^{\prime} / \mathrm{G}\right)$-expansion technique is presented in [27].

We analyzed the time fractional Gardner equation. The Gardner equation is an advantageous example for the definition of interior solitary waves in shallow water, while Buckmaster's equation is applied in thin viscous fluid sheet flows. Gardner equation is extensively used in several physics and engineering problems consisting of fluid physics, chemical engineering, and quantum discipline principle. The equation presents outstanding function in ocean waves. In shallow seas Gardner equation defines internal solitary waves. This equation scrutinized within the literature because it's far used to model a diffusion of nonlinear phenomena. Gardner equation is solved by way of using F-expansion in [28] and has been generally examined by several other methods [29-31].

The Fornberg-Whitham equation has been found to require peakon results as a simulation for limiting wave heights as well as the frequency of wave breaks. In fractional calculus has gained considerable significance and popularity, primarily because of its well-shown applications in a wide range of apparently disparate areas of engineering and science. Fractional Fornberg-Whitham equation is solved by using fractional variational iteration method in [32]. Kumar [33] to resolve the fractional Fornberg-Whitham equation via Laplace transform. Besides, the Laplace Adomian decomposition approach is likewise used in [34]. Many scholars have therefore researched the fractional extensions of the FornbergWhitham model for the Caputo fractional-order derivative [35-37].

\section{Basic concept for fractional calculus}

Some primary definitions of fractional calculus are recalled which can be practiced in the calculation. It is famous that there are precise definitions of fractional Integral and fractional derivatives, which includes, GrünwaldLetnikov, Riesz, Riemann-Liouville, Caputo, Hadamard and Erdélyi-Kober and lots of others [38-40].

Definition 2.1 The fractional integral operator of RL of order $\alpha \geq 0$ of a function $f \in C \mu, \mu \geq-1$ is defined as

$$
\begin{gathered}
J^{\alpha} f(x)=\frac{1}{\Gamma(\alpha)} \int_{a}^{x}(x-v)^{\alpha-1} d v, \quad \alpha>0, \quad x>0 \\
J^{0} f(x)=f(x) .
\end{gathered}
$$

Definition 2.2 The Caputo's fractional derivative is [41]

$$
\begin{aligned}
& D^{\alpha} t^{\xi}= \begin{cases}\frac{\Gamma(\xi+1)}{\Gamma(\xi+1-\alpha)} t^{\xi-\alpha}, & \xi \in \mathrm{N}_{0}, \xi \geq\lceil\alpha\rceil \\
0, & \xi \in \mathrm{N}_{0}, \xi<\lceil\alpha\rceil,\end{cases} \\
& D^{\alpha} c=0, c \in \Re .
\end{aligned}
$$

Definition 2.3 Let $f$ be an $n$ time differentiable at $x$ then the conformable fractional derivative is well-defined by [41]

$$
\begin{aligned}
& \left(T_{\alpha} f\right)(x)=\lim _{\varepsilon \rightarrow 0} \frac{f^{([\alpha]-1)}\left(x+\varepsilon x^{[\alpha]-\alpha}\right)-f^{([\alpha]-1)}(x)}{\varepsilon}, \\
& x>0, \quad \alpha \in(0,1] .
\end{aligned}
$$

If the above limit exists, then $f$ is called $\alpha$ differentiable. Let $\alpha \in(0,1]$ and $f$, $g$ be $\alpha$-differentiable at a point $x>0$, then $T_{\alpha}$ satisfies the following properties:

1.

$$
T_{\alpha}(a f+b g)=a T_{\alpha}(f)+b T_{\alpha}(g), \text { for all } a, b \in \Re
$$

2.

$$
T_{\alpha}\left(x^{p}\right)=p x^{p-\alpha}, \text { for all } p \in \Re
$$

3. $T_{\alpha}(\lambda)=0$, for all constant functions $f(x)=\lambda$

4.

$$
T_{\alpha}(f g)=f T_{\alpha}(g)+g T_{\alpha}(f)
$$

5.

$$
T_{\alpha}(f / g)=\left\{g T_{\alpha}(f)-f T_{\alpha}(g)\right\} / g^{2}
$$

6. If, in addition, $f$ is differentiable $T_{\alpha}(f)(x)=$ $x^{1-\alpha} \frac{d f}{d x}(x)$

\section{Analysis of conformable fractional variational iteration method (CFVIM)}

Firstly the conformable fractional variational iteration method is discussed for the solution of the following nonlinear time fractional partial differential equation

$T_{t}^{\alpha} w(x, t)+L(w(x, t))+N(w(x, t))=h(x, t), n<\alpha \leq n+1$,

where $L$ and $N$ is linear and non-linear operator respectively, $\mathrm{h}(\mathrm{x})$ is source term and $T_{t}^{\alpha}$ is conformable fractional derivative of order $\alpha$. To solve differential Equation (5) via conformable fractional variational iteration method, write the differential Eq. (5) in the form,

$$
t^{[\alpha\rceil-\alpha} \frac{\partial^{([\alpha])} w(x, t)}{\partial t^{(\alpha \alpha)}}+L(w(x, t))+N(w(x, t))=h(x, t) .
$$


As in conformable fractional variational iteration method, the correction functional for Eq. (6) can be

$$
\begin{aligned}
& w_{n+1}(x, t)=w_{n}(x, t)+\int_{0}^{t} \mu(\tau)\left(\tau^{\lceil\alpha\rceil-\alpha} \frac{\partial^{([\alpha])} w_{n}(x, \tau)}{\partial \tau^{(\alpha \alpha)}}\right. \\
& \left.+L\left(w_{n}(x, \tau)\right)+N\left(\tilde{w}_{n}(x, \tau)\right)-h(x, \tau)\right) d \tau .
\end{aligned}
$$

Finally, the solution is

$$
w(x, t)=\lim _{n \rightarrow \infty} w_{n}(x, t) .
$$

\section{Analysis of conformable fractional reduced differential transform method (CFRDTM)}

Let $w(x, t)$ is continuous and analytic with respect to time $t$ and space $x$. conformable fractional reduce differential transform of $w(x, t)$ is defined as

$$
W_{k}^{\alpha}(x)=\frac{1}{\alpha^{k} k !}\left[T_{t}^{\alpha(k)} w\right]_{t=t_{0}} .
$$

Where some $0<\alpha \leq 1, \alpha$ is a parameter labeling the order of CFD, $T_{t}{ }^{\alpha(k)} w=\underbrace{T_{t}{ }^{\alpha} T_{t}{ }^{\alpha} T_{t}{ }^{\alpha} \ldots T_{t}{ }^{\alpha}}_{k \text {-times }} w(x, t)$.

The inverse conformable fractional reduce differential transform of $W_{k}^{\alpha}(x)$ is defined as

$w(x, t)=\sum_{k=0}^{\infty} W_{k}^{\alpha}(x)\left(t-t_{0}\right)^{\alpha k}=\sum_{k=0}^{\infty} \frac{1}{\alpha^{k} k !}\left[T_{t}^{\alpha(k)} w\right]_{t=t_{0}}\left(t-t_{0}\right)^{\alpha k}$

For integer order derivatives the initial conditions for conformable fractional reduce differential transform are defined as

$$
\begin{aligned}
& W_{k}^{\alpha}(x)= \begin{cases}\frac{1}{(\alpha k) !}\left[\frac{\partial^{\alpha k}}{\partial t^{\alpha k}} w(x, t)\right] & \text { if } \alpha k \in \mathrm{Z}^{+} \\
0 & \text { if } \alpha k \notin \mathrm{Z}^{+}\end{cases} \\
& \text {for } k=0,1,2, \ldots,\left(\frac{n}{\alpha}-1\right)
\end{aligned}
$$

where $n$ is the order of conformable fractional partial differential equation.

By considering $W_{k}^{\alpha}(x)=f(x)$ as the initial condition

$$
w(x, 0)=f(x) .
$$

and

$$
\tilde{w}_{n}(x, t)=\sum_{k=0}^{n} W_{k}^{\alpha}(x) t^{\alpha k} .
$$

The solution is written as

$$
w(x, t)=\lim _{n \rightarrow \infty} \tilde{w}_{n}(x, t) .
$$

\section{Numerical applications}

\subsection{The foam drainage equation}

$$
T_{t}^{\alpha} w=\frac{1}{2} w w_{x x}-2 w^{2} w_{x}+\left(w_{x}\right)^{2},
$$

subject to condition

$$
w(x, 0)=-\sqrt{c} \tanh (\sqrt{c} x) .
$$

According to conformable fractional variational iteration method, the correctional functional of Eq. (15) is read as

$$
\begin{aligned}
& w_{n+1}(x, t)=w_{n}(x, t) \\
& +\int_{0}^{t} \mu\left(\tau^{1-\alpha}\left(w_{n \tau}\right)-\frac{1}{2} w_{n} w_{n x x}+2 w_{n}{ }^{2} w_{n x}-\left(w_{n x}\right)^{2}\right) d \tau
\end{aligned}
$$

$$
\begin{aligned}
& w_{n+1}(x, t)=w_{n}(x, t) \\
& -\int_{0}^{t}\left(\tau^{1-\alpha}\left(w_{n \tau}\right)-\frac{1}{2} w_{n} w_{n x x}+2 w_{n}{ }^{2} w_{n x}-\left(w_{n x}\right)^{2}\right) d \tau
\end{aligned}
$$

Consequently, we get

$$
w_{0}(x, t)=-\sqrt{c} \tanh (\sqrt{c} x),
$$

$$
\begin{aligned}
& w_{1}(x, t)=-\sqrt{c} \tanh (\sqrt{c} x)-\frac{c^{2} t^{2-\alpha} \operatorname{Sech}(\sqrt{c} x)^{2}}{\alpha-2}, \\
& w_{2}(x, t)=-\frac{2}{3} c^{5} t^{3} \operatorname{Sech}(\sqrt{c} x)^{4}-\sqrt{c} \operatorname{Tanh}(\sqrt{c} x) \\
& +c^{5} t^{3} \operatorname{Sech}(\sqrt{c} x)^{4}\left(1+c^{\frac{3}{2}} t \operatorname{Tanh}(\sqrt{c} x)\right) \\
& +\frac{1}{\alpha-2}\left(c ^ { 2 } t \operatorname { S e c h } ( \sqrt { c } x ) ^ { 2 } \left(t^{1-\alpha}+(\alpha-2)\right.\right. \\
& \left.\left.\left(2+c^{\frac{3}{2}} t \operatorname{Tanh}(\sqrt{c} x)\right)\right)\right),
\end{aligned}
$$

Now, by applying conformable fractional reduce differential transform on Eq. (15) and (16), we get

$$
W_{0}^{\alpha}(x)=-\sqrt{c} \tanh (\sqrt{c} x),
$$

$$
W_{2}^{\alpha}(x)=\frac{1}{\alpha^{2}}\left(c^{\frac{7}{2}} \operatorname{Sech}(\sqrt{c} x)^{2} \operatorname{Tanh}(\sqrt{c} x)\right),
$$

$$
W_{3}^{\alpha}(x)=\frac{c^{5}}{3 \alpha^{3}}\left(-2+\operatorname{Cosh}(2 \sqrt{c} x) \operatorname{Sech}(\sqrt{c} x)^{4}\right),
$$


The series solution is

$$
\begin{gathered}
\tilde{w}(x, t)=\sum_{k=0}^{n} W_{k}^{\alpha}(x) t^{k \alpha}, \\
\tilde{w}(x, t)=W_{0}^{\alpha}+W_{1}^{\alpha} t+W_{2}^{\alpha} t^{2}+W_{3}^{\alpha} t^{3}+\ldots, \\
\tilde{w}(x, t)=-\sqrt{c} \tanh (\sqrt{c} x)+\frac{1}{\alpha}\left(c^{2} \operatorname{Sech}(\sqrt{c} x)^{2}\right) t+\frac{1}{\alpha^{2}}\left(c^{\frac{7}{2}} \operatorname{Sech}(\sqrt{c} x)^{2} \operatorname{Tanh}(\sqrt{c} x)\right) t^{2} \\
+\frac{c^{5}}{3 \alpha^{3}}\left(-2+\operatorname{Cosh}(2 \sqrt{c} x) \operatorname{Sech}(\sqrt{c} x)^{4}\right) t^{3}+\ldots,
\end{gathered}
$$

The exact solution of Eq. (15) is

$$
w(x, t)=\left\{\begin{array}{c}
-\sqrt{c} \tanh (\sqrt{c}(x-c t)) ; \quad x \leq c t \\
0 ; \quad x>c t
\end{array}\right.
$$

\begin{tabular}{|c|c|c|c|c|c|c|}
\hline \multirow[t]{2}{*}{$x$} & \multirow[t]{2}{*}{$\mathrm{t}$} & \multirow[t]{2}{*}{ CFRDTM } & \multirow[t]{2}{*}{ CFVIM } & \multirow[t]{2}{*}{ Exact Sol } & \multicolumn{2}{|c|}{ Absolute Error } \\
\hline & & & & & CFRDTM & CFVIM \\
\hline \multirow{4}{*}{-0.2} & 0.01 & 0.206967 & 0.206967 & 0.206966 & $1.2631 \times 10^{-5}$ & $1.2011 \times 10^{-6}$ \\
\hline & 000 & 02 & $00 ?$ & 0100236 & $2.0421 \times 10^{-7}$ & $1.1052 \times 10^{-8}$ \\
\hline & & & & & $2.0531 \times 10^{-7}$ & $1.5014 \times 10^{-9}$ \\
\hline & 0.0001 & 0.197471 & 0.197471 & 0.197471 & & \\
\hline \multirow{4}{*}{-0.5} & 0.01 & 0.469945 & 0.469945 & 0.469945 & $2.2421 \times 10^{-8}$ & $1.2540 \times 10^{-10}$ \\
\hline & $0 \cap 01$ & בח & 0 & בח 0260 & $2.5321 \times 10^{-8}$ & $1.4251 \times 10^{-10}$ \\
\hline & & & & & $2.6173 \times 10^{-8}$ & $2.2530 \times 10^{-10}$ \\
\hline & 0.0001 & 0.462196 & 0.462196 & 0.462196 & & \\
\hline \multirow{4}{*}{-0.7} & 0.01 & 0.610677 & 0.610677 & 0.610677 & $1.2003 \times 10^{-8}$ & $1.3046 \times 10^{-10}$ \\
\hline & 0001 & ר 00500 & 7605006 & ר 00500 & $1.5631 \times 10^{-8}$ & $1.7613 \times 10^{-10}$ \\
\hline & & & & & $1.8641 \times 10^{-8}$ & $1.9141 \times 10^{-10}$ \\
\hline & 0.0001 & 0.604431 & 0.604431 & 0.604431 & & \\
\hline \multirow{4}{*}{-0.9} & 0.01 & 0.721132 & 0.721132 & 0.721132 & $1.5042 \times 10^{-8}$ & $2.2931 \times 10^{-10}$ \\
\hline & 001 & 071678 & 071678 & 0 & $1.9402 \times 10^{-8}$ & $2.4641 \times 10^{-10}$ \\
\hline & 0.001 & 0.110784 & 0.18074 & 0.110764 & $2.0047 \times 10^{-8}$ & $3.0021 \times 10^{-10}$ \\
\hline & 0.0001 & 0.716347 & 0.716347 & 0.716347 & & \\
\hline \multirow{4}{*}{-1} & 0.01 & 0.765762 & 0.765762 & 0.765762 & $3.0042 \times 10^{-8}$ & $4.0001 \times 10^{-10}$ \\
\hline & 0.001 & 0762014 & 0762014 & $076201 /$ & $3.9442 \times 10^{-8}$ & $4.6851 \times 10^{-10}$ \\
\hline & & & & & $4.0723 \times 10^{-8}$ & $5.0031 \times 10^{-10}$ \\
\hline & 0.0001 & 0.761636 & 0.761636 & 0.761636 & & \\
\hline
\end{tabular}

Here $\mathrm{c}$ is the speed of wave.

Table 1: Numerical analysis of solution Eq. (21), (28) and exact solution (29) for example 1 at $\mathrm{c}=1$ and $\alpha=1$ 
Figure1

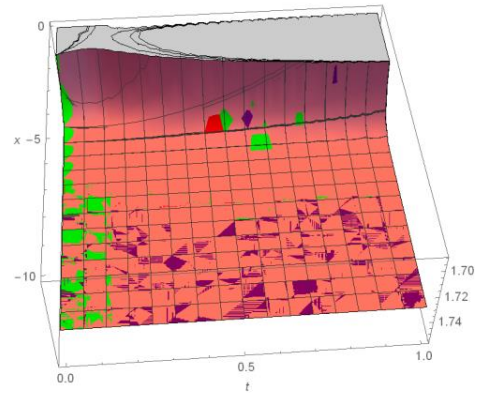

$\alpha=0.2 \square \alpha=0.5 \square \alpha=0.8 \square \alpha=1$

(a)

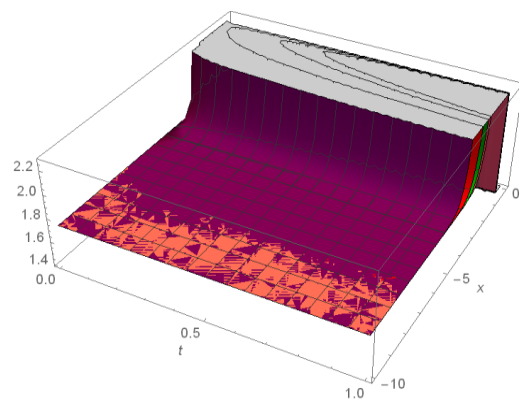

$\alpha=0.2 \square \alpha=0.5 \square \alpha=0.8 \square \alpha=1$

(b)

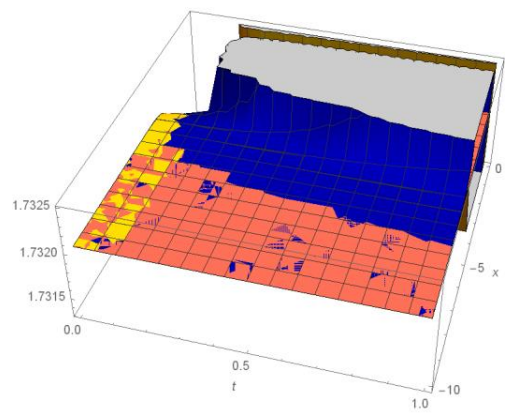

CFRDTM App sol $\square$ CVIM App sol $\square$ Exact sol

(c)

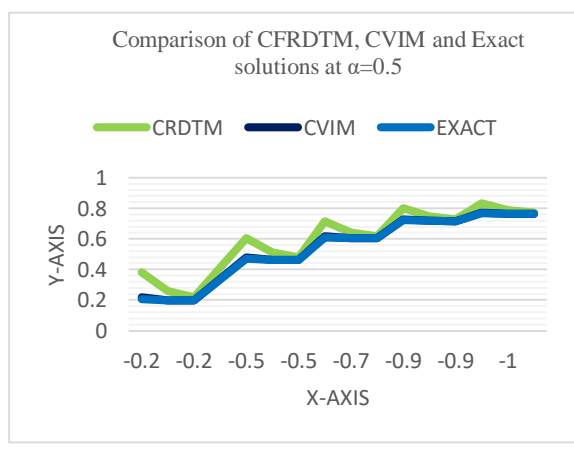

(d)
Comparison of CFRDTM, CVIM and Exact solutions at $\alpha=1$

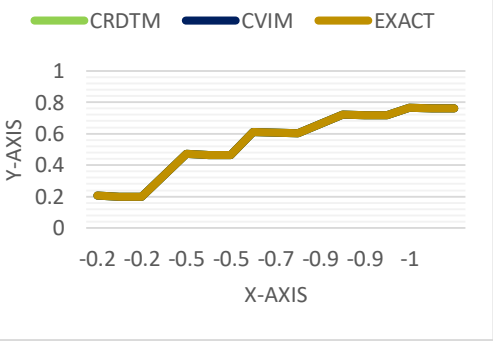

(e)

Figure 1: Approximate solution for the foam drainage model Eq. (15) for different values of $\alpha$ with fixed values $0 \leq t \leq 1,-10 \leq x \leq 0$. 


\subsection{Time-fractional Gardner equation}

$$
T_{t}^{\alpha} u+6\left(u-\varepsilon^{2} u^{2}\right) u_{x}+u_{x x x}=0
$$

with the initial condition

$$
w(x, 0)=\frac{1}{2}+\frac{1}{2} \tanh \left[\frac{x}{2}\right]
$$

For $\varepsilon=1$, (30) will reduce into

$$
T_{t}^{\alpha} w+6\left(w-w^{2}\right) w_{x}+w_{x x x}=0
$$

According to conformable fractional variational iteration method, the correctional functional of Eq. (32) is

$$
\begin{gathered}
w_{n+1}(x, t)=w_{n}(x, t)+\int_{0}^{t} \mu\left(\tau^{1-\alpha}\left(w_{n \tau}\right)+6 w_{n} w_{n x}-6 w_{n}{ }^{2} w_{n x}-w_{n x x x}\right) d \tau \\
w_{n+1}(x, t)=w_{n}(x, t)-\int_{0}^{t}\left(\tau^{1-\alpha}\left(w_{n \tau}\right)+6 w_{n} w_{n x}-6 w_{n}{ }^{2} w_{n x}-w_{n x x x}\right) d \tau
\end{gathered}
$$

Consequently, we get

$$
w_{0}(x, t)=\frac{1}{2}+\frac{1}{2} \tanh \left[\frac{x}{2}\right]
$$

$$
w_{1}(x, t)=\frac{1}{8}\left(4-9 t \operatorname{Sech}\left(\frac{x}{2}\right)^{2}+t \operatorname{Sech}\left(\frac{x}{2}\right)^{4}-96 t \operatorname{Csch}(x)^{3} \operatorname{Sinh}\left(\frac{x}{2}\right)^{4}-80 t \operatorname{Csch}(x)^{4} \operatorname{Sinh}\left(\frac{x}{2}\right)^{6}+4 \operatorname{Tanh}\left(\frac{x}{2}\right)\right)
$$

$$
\begin{aligned}
& w_{2}(x, t)=-\frac{1}{8} t \operatorname{Sech}\left(\frac{x}{2}\right)^{4}(1+7 \operatorname{Cos}(x)+6 \operatorname{Sin}(x))+\frac{1}{2}\left(1+\operatorname{Tanh}\left(\frac{x}{2}\right)\right)+\frac{1}{98304} t \operatorname{Sech}\left(\frac{x}{2}\right)^{12}\left(1+\operatorname{Tanh}\left(\frac{x}{2}\right)\right) \\
& \left(\begin{array}{l}
-64512+36960 t-8012 t^{3}-\frac{23688}{-2+\alpha} t^{1-\alpha}+4\left(-27738+20265 t-4064 t^{2}\right. \\
\left.+1356 t^{3}-\frac{9936}{-2+\alpha} t^{1-\alpha}\right) \operatorname{Cosh}(x)+\left(\begin{array}{c}
-70020+74694 t-9712 t^{2} \\
+15924 t^{3}-\frac{23220}{-2+\alpha} t^{1-\alpha}
\end{array}\right) \operatorname{Cosh}(2 x) \\
-31620 \operatorname{Cosh}(3 x)+39594 t \operatorname{Cosh}(3 x)-128 t^{2} \operatorname{Cosh}(3 x)-6576 t^{3} \operatorname{Cosh}(3 x) \\
-\frac{9936}{-2+\alpha} t^{1-\alpha} \operatorname{Cosh}(3 x)-9696 \operatorname{Cosh}(4 x)+9648 t \operatorname{Cosh}(4 x)+3 t^{3} \operatorname{Cosh}(4 x) \\
-\frac{2232}{-2+\alpha} t^{1-\alpha} \operatorname{Cosh}(4 x)-1812 \operatorname{Cosh}(5 x)+690 t \operatorname{Cosh}(5 x)-\frac{288}{-2+\alpha} t^{1-\alpha} \operatorname{Cosh}(5 x) \\
-156 \operatorname{Cosh}(6 x)+42 t \operatorname{Cosh}(6 x)-\frac{12}{-2+\alpha} t^{1-\alpha} \operatorname{Cosh}(6 x)-1944 \operatorname{Sinh}(x) \\
-11336 t^{2} \operatorname{Sinh}(x)+4170 t^{3} \operatorname{Sinh}(x)-\frac{4464}{-2+\alpha} t^{1-\alpha} \operatorname{Sinh}(x)-2052 \operatorname{Sinh}(2 x) \\
17118 t \operatorname{Sinh}(2 x)-8360 t^{2} \operatorname{Sinh}(2 x)+15510 t^{3} \operatorname{Sinh}(2 x)-\frac{4932}{-2+\alpha} t^{1-\alpha} \operatorname{Sinh}(2 x) \\
-948 \operatorname{Sinh}(3 x)+7962 t \operatorname{Sinh}(3 x)+24 t^{2} \operatorname{Sinh}(3 x)-6606 t^{3} \operatorname{Sinh}(3 x) \\
-\frac{2568}{-2+\alpha} t^{1-\alpha} \operatorname{Sinh}(3 x)-144 \operatorname{Sinh}(4 x)+1488 t \operatorname{Sinh}(4 x)+1364 t^{2} \operatorname{Sinh}(4 x) \\
-3 t^{3} \operatorname{Sinh}(4 x)-\frac{624}{-2+\alpha} t^{1-\alpha} \operatorname{Sinh}(4 x)+36 \operatorname{Sinh}(5 x)-66 t \operatorname{Sinh}(5 x) \\
-\frac{24}{-2+\alpha} t^{1-\alpha} \operatorname{Sinh}(5 x)+12 \operatorname{Sinh}(6 x)-42 t \operatorname{Sinh}(6 x)+\frac{12}{-2+\alpha} t^{1-\alpha} \operatorname{Sinh}(6 x)
\end{array}\right)
\end{aligned}
$$

And so on.

Now, by applying conformable fractional reduce differential transform on Eq. (32) and (31), we get

$$
\begin{gathered}
W_{0}^{\alpha}(x)=\frac{1}{2}+\frac{1}{2} \operatorname{Tanh}\left(\frac{x}{2}\right), \\
W_{1}^{\alpha}(x)=\frac{1}{8 \alpha}\left(\operatorname{Sech}\left(\frac{x}{2}\right)^{4}\right)(-1+5 \operatorname{Cosh}(x)+6 \operatorname{Sinh}(x)), \\
W_{2}^{\alpha}(x)=-\frac{1}{128 \alpha^{2}}\left(\operatorname{Sech}\left(\frac{x}{2}\right)^{7}\right)\left(\begin{array}{l}
168 \operatorname{Cosh}\left(\frac{x}{2}\right)-420 \operatorname{Cosh}\left(\frac{3 x}{2}\right)+60 \operatorname{Cosh}\left(\frac{5 x}{2}\right) \\
-190 \operatorname{Sinh}\left(\frac{x}{2}\right)-417 \operatorname{Sinh}\left(\frac{3 x}{2}\right)+61 \operatorname{Sinh}\left(\frac{5 x}{2}\right)
\end{array}\right),
\end{gathered}
$$




$$
W_{3}^{\alpha}(x)=-\frac{1}{3072 \alpha^{3}}\left(\operatorname{Sech}\left(\frac{x}{2}\right)^{10}\right)\left(\begin{array}{l}
-48737+3686 \operatorname{Cosh}(x)+59336 \operatorname{Cosh}(2 x) \\
-21278 \operatorname{Cosh}(3 x)+665 \operatorname{Cosh}(4 x)+ \\
7909 \operatorname{Sinh}(x)+49500 \operatorname{Sinh}(2 x) \\
-21276 \operatorname{Sinh}(3 x)+666 \operatorname{Sinh}(4 x)
\end{array}\right),
$$

The series solution is

$$
\begin{aligned}
& \tilde{w}(x, t)=\frac{1}{2}+\frac{1}{2} \operatorname{Tanh}\left(\frac{x}{2}\right)+\frac{1}{8 \alpha}\left(\operatorname{Sech}\left(\frac{x}{2}\right)^{4}\right)(-1+5 \operatorname{Cosh}(x)+6 \operatorname{Sinh}(x)) t+ \\
& -\frac{1}{128 \alpha^{2}}\left(\operatorname{Sech}\left(\frac{x}{2}\right)^{7}\right)\left(\begin{array}{l}
168 \operatorname{Cosh}\left(\frac{x}{2}\right)-420 \operatorname{Cosh}\left(\frac{3 x}{2}\right)+60 \operatorname{Cosh}\left(\frac{5 x}{2}\right)-190 \operatorname{Sinh}\left(\frac{x}{2}\right)- \\
417 \operatorname{Sinh}\left(\frac{3 x}{2}\right)+61 \operatorname{Sinh}\left(\frac{5 x}{2}\right)
\end{array}\right) t^{2}+ \\
& -\frac{1}{3072 \alpha^{3}}\left(\operatorname{Sech}\left(\frac{x}{2}\right)^{10}\right)\left(\begin{array}{l}
-48737+3686 \operatorname{Cosh}(x)+59336 \operatorname{Cosh}(2 x)-21278 \operatorname{Cosh}(3 x)+ \\
665 \operatorname{Cosh}(4 x)+7909 \operatorname{Sinh}(x)+49500 \operatorname{Sinh}(2 x)-21276 \operatorname{Sinh}(3 x) \\
+666 \operatorname{Sinh}(4 x)
\end{array}\right) t^{3}+\ldots,
\end{aligned}
$$

The exact solution of Eq. (30) is

\begin{tabular}{|c|c|c|c|c|c|c|}
\hline \multirow[t]{2}{*}{$x$} & \multirow[t]{2}{*}{$\mathrm{t}$} & \multirow[t]{2}{*}{ CFRDTM } & \multirow[t]{2}{*}{ CFVIM } & \multirow[t]{2}{*}{ Exact Sol } & \multicolumn{2}{|c|}{ Absolute Error } \\
\hline & & & & & CFRDTM & CFVIM \\
\hline 0.2 & $\begin{array}{c}0.0001 \\
0.00001 \\
0.000001\end{array}$ & $\begin{array}{l}0.549899 \\
0.549841 \\
0.549835\end{array}$ & $\begin{array}{l}0.549509 \\
0.549801 \\
0.549831\end{array}$ & $\begin{array}{l}0.549809 \\
0.549832 \\
0.549834\end{array}$ & $\begin{array}{l}9.0131 \times 10^{-5} \\
9.0421 \times 10^{-6} \\
1.0531 \times 10^{-6}\end{array}$ & $\begin{array}{l}3.0520 \times 10^{-4} \\
3.1052 \times 10^{-5} \\
3.0114 \times 10^{-6}\end{array}$ \\
\hline 0.5 & $\begin{array}{c}0.0001 \\
0.00001 \\
0.000001\end{array}$ & $\begin{array}{l}0.622545 \\
0.622468 \\
0.622460\end{array}$ & $\begin{array}{l}0.622086 \\
0.622422 \\
0.622456\end{array}$ & $\begin{array}{l}0.622436 \\
0.622457 \\
0.622459\end{array}$ & $\begin{array}{l}1.0921 \times 10^{-4} \\
1.1032 \times 10^{-5} \\
1.0013 \times 10^{-6}\end{array}$ & $\begin{array}{l}3.5254 \times 10^{-4} \\
3.5421 \times 10^{-5} \\
3.6253 \times 10^{-6}\end{array}$ \\
\hline 0.7 & $\begin{array}{c}0.0001 \\
0.00001 \\
0.000001\end{array}$ & $\begin{array}{l}0.668284 \\
0.668197 \\
0.668189\end{array}$ & $\begin{array}{l}0.667784 \\
0.668147 \\
0.668184\end{array}$ & $\begin{array}{l}0.668166 \\
0.668186 \\
0.668188\end{array}$ & $\begin{array}{l}1.1803 \times 10^{-4} \\
1.1245 \times 10^{-5} \\
1.0021 \times 10^{-6}\end{array}$ & $\begin{array}{l}4.0231 \times 10^{-4} \\
3.9214 \times 10^{-5} \\
4.0241 \times 10^{-6}\end{array}$ \\
\hline 0.9 & $\begin{array}{c}0.0001 \\
0.00001 \\
0.000001\end{array}$ & $\begin{array}{l}0.711054 \\
0.710960 \\
0.710951\end{array}$ & $\begin{array}{l}0.710517 \\
0.710906 \\
0.710945\end{array}$ & $\begin{array}{l}0.710929 \\
0.710947 \\
0.710949\end{array}$ & $\begin{array}{l}1.2504 \times 10^{-4} \\
1.3110 \times 10^{-5} \\
2.0047 \times 10^{-6}\end{array}$ & $\begin{array}{l}4.1234 \times 10^{-4} \\
4.1064 \times 10^{-5} \\
4.2021 \times 10^{-6}\end{array}$ \\
\hline 1 & $\begin{array}{c}0.0001 \\
0.00001 \\
0.000001\end{array}$ & $\begin{array}{l}0.731165 \\
0.731069 \\
0.731060\end{array}$ & $\begin{array}{l}0.730613 \\
0.731014 \\
0.731054\end{array}$ & $\begin{array}{l}0.731039 \\
0.731057 \\
0.731058\end{array}$ & $\begin{array}{l}1.2604 \times 10^{-4} \\
5.5242 \times 10^{-4} \\
2.0123 \times 10^{-6}\end{array}$ & $\begin{array}{l}4.2601 \times 10^{-4} \\
2.5129 \times 10^{-5} \\
4.0062 \times 10^{-6}\end{array}$ \\
\hline
\end{tabular}

$$
u(x, t)=\frac{1}{2}+\frac{1}{2} \tanh \left[\frac{x-t}{2}\right]
$$

Table 2: Numerical analysis of solution Eq. (37), (42) and exact solution (43) for example 2 at $\alpha=1$

\subsection{Time-fractional Fornberg-Whitham equation}

$$
D_{t}^{\alpha} w-w_{x x t}+w_{x}=w w_{x x x}-w w_{x}+3 w_{x} w_{x x}, t>0, \alpha>0,
$$




\section{Figure2}

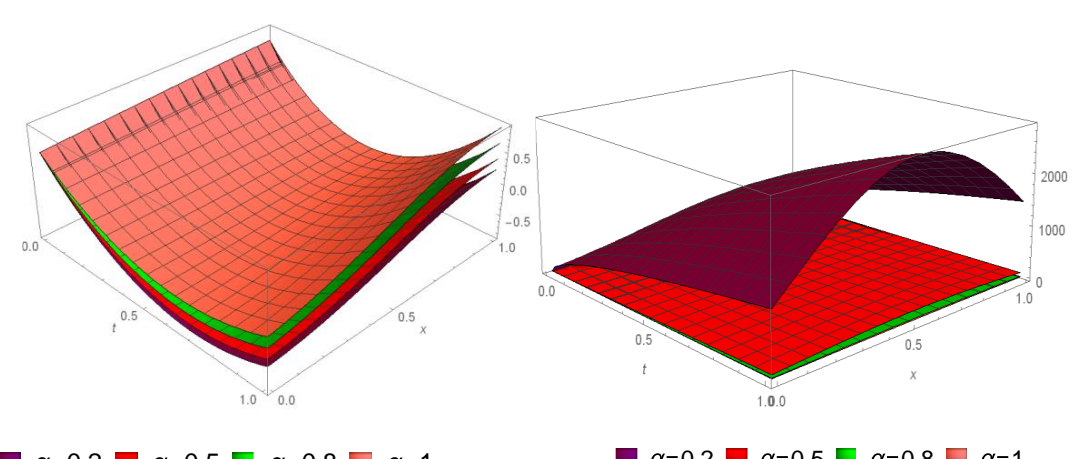

$\alpha=0.2 \square \alpha=0.5 \square \alpha=0.8 \square \alpha=1$

(a)

(b)

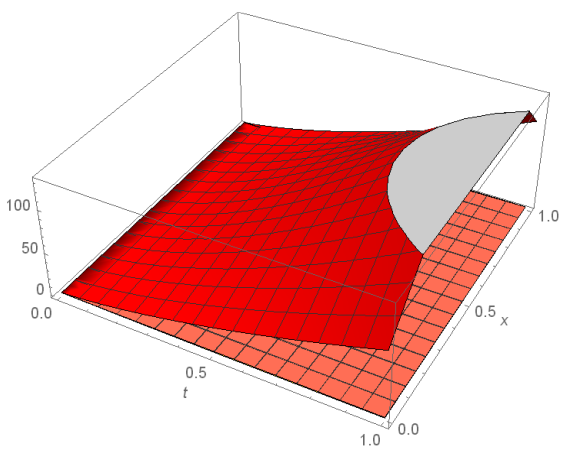

CFRDTM App sol $\square$ CVIM App sol $\square$ Exact sol

(c)

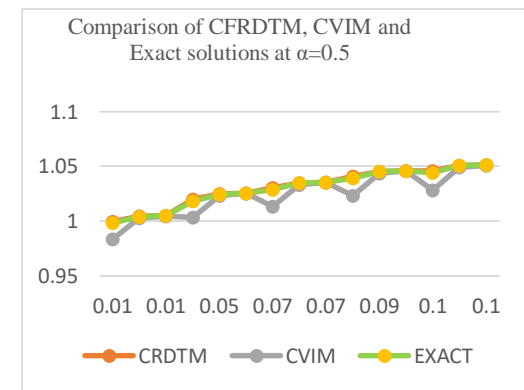

(d)

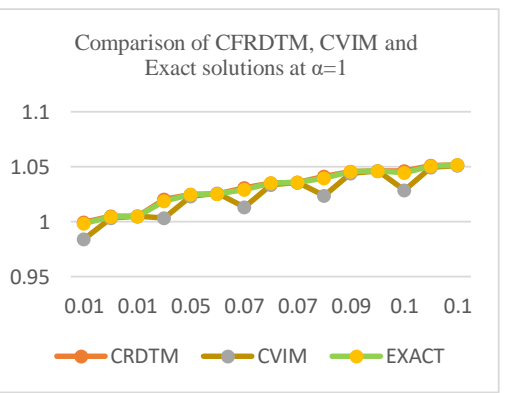

(e)

Figure 2: Approximate solution for the time-fractional Gardner Eq. (32) for different values of $\alpha$ with fixed values $0 \leq \mathrm{t} \leq 1,0 \leq \mathrm{x} \leq 1$. 
subject to initial condition

$$
w(x, 0)=e^{\frac{1}{2} x} .
$$

According to conformable fractional variational iteration method, the correctional functional of Eq. (44) is

$$
\begin{aligned}
& w_{n+1}(x, t)=w_{n}(x, t)+\int_{0}^{t} \mu\left(\tau^{1-\alpha}\left(w_{n \tau}\right)-w_{n x x t}+w_{n x}-w_{n} w_{n x x x}+w_{n} w_{n x}+3 w_{n x} w_{n x x}\right) d \tau \\
& w_{n+1}(x, t)=w_{n}(x, t)-\int_{0}^{t}\left(\tau^{1-\alpha}\left(w_{n \tau}\right)-w_{n x x t}+w_{n x}-w_{n} w_{n x x x}+w_{n} w_{n x}+3 w_{n x} w_{n x x}\right) d \tau
\end{aligned}
$$

Consequently, we get

$$
\begin{gathered}
w_{0}(x, t)=e^{\frac{1}{2} x}, \\
w_{1}(x, t)=e^{\frac{1}{2} x}-\left(\frac{e^{\frac{1}{2} x}}{2}+\frac{3 e^{x}}{4}\right) t, \\
w_{2}(x, t)=\frac{1}{64(-2+\alpha)}\left(e^{\frac{1}{2} x} t^{-\alpha}\left(\begin{array}{c}
-16\left(2+3 e^{\frac{1}{2} x}\right) t^{2}- \\
t^{\alpha}\left(-64+t\left(\begin{array}{c}
-8(-9+t)+4 e^{\frac{1}{2} x}(-6+t)^{2} \\
+21 e^{x}(-3+t) t+36 e^{\frac{3}{2}} x t^{2}
\end{array}\right)\right)(-2+\alpha)
\end{array}\right)\right),
\end{gathered}
$$

And so on.

Now, by applying conformable fractional reduce differential transform on Eq. (44) and (45), we get

$$
\begin{gathered}
W_{0}^{\alpha}(x)=e^{\frac{1}{2} x}, \\
W_{1}^{\alpha}(x)=-\frac{1}{2 \alpha}\left(e^{\frac{1}{2} x}\right), \\
W_{2}^{\alpha}(x)=\frac{1}{8 \alpha^{2}}\left(e^{\frac{1}{2} x}\right), \\
W_{3}^{\alpha}(x)=-\frac{1}{48 \alpha^{3}}\left(e^{\frac{1}{2} x}\right),
\end{gathered}
$$

The series solution is

$$
\tilde{w}(x, t)=e^{\frac{1}{2} x}+\frac{1}{2 \alpha}\left(e^{\frac{1}{2} x}\right) t+\frac{1}{8 \alpha^{2}}\left(e^{\frac{1}{2} x}\right) t^{2}+\frac{1}{48 \alpha^{3}}\left(e^{\frac{1}{2} x}\right) t^{3}+\ldots,
$$

The closed form solution of Eq. (44) is

$$
w(x, t)=e^{\frac{1}{2}\left|x-\frac{4}{3} t\right|} .
$$

\section{Results and discussions}

From the above results, Figure 1 (a) represents the physical behavior of solution equation (21) by CFVIM or different values of $\alpha$ with fixed values $0 \leq \mathrm{t} \leq 1,-10 \leq \mathrm{x} \leq 0$. (b) Shows physical behavior of the solution using CFRDTM of equation (28) for different values of $\alpha$ with fixed values $0 \leq \mathrm{t} \leq 1,-10 \leq \mathrm{x} \leq 0$. (c) Depicts the comparison of CFVIM and CFRDTM with exact solution for $\alpha=1$ with fixed values $0 \leq \mathrm{t} \leq 1,-10 \leq \mathrm{x} \leq 0$. (d) Depicts the comparison of CFVIM and CFRDTM with exact solution with $\alpha=0.5$ for different values $\mathrm{x}$ and t. (e) Illustrations the comparison of CFVIM and CFRDTM with exact solution with for different values $\mathrm{x}$ and $\mathrm{t}$ for $\alpha=1$. Figure 2 (a) shows the physical behavior of CFVIM's 


\section{Figure3}

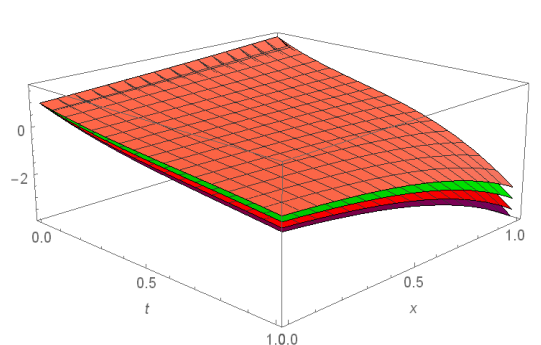

$\alpha=0.2 \square \alpha=0.5 \square \alpha=0.8 \square \alpha=1$

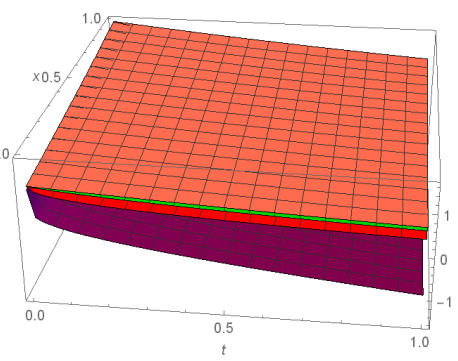

$\square \alpha=0.2 \square \alpha=0.5 \square \alpha=0.8 \square \alpha=1$

(a)

(b)

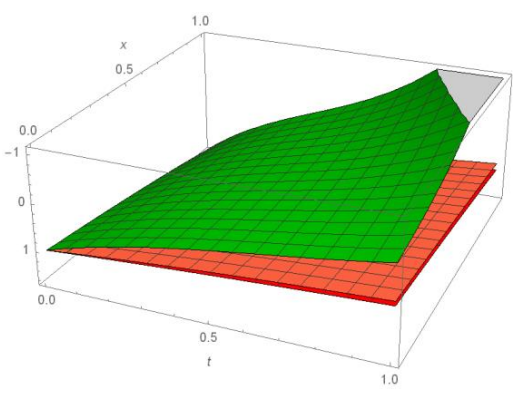

$\square$ CFRDTM App sol $\square$ CVIM App sol $\square$ Exact sol

(c)

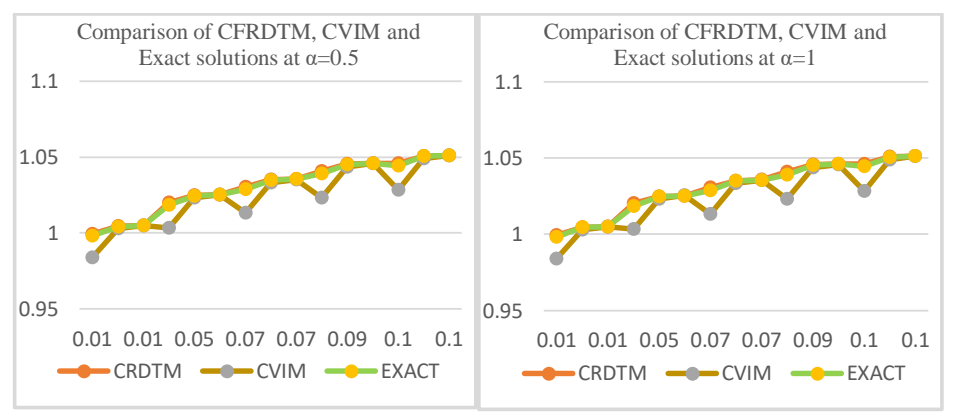

(d)

(e)

Figure 3: Approximate solution for the time-fractional Fornberg-Whitham Eq. (44) for different values of $\alpha$ with fixed values $0 \leq \mathrm{t} \leq 1,0 \leq \mathrm{x}$ $\leq 1$. 
Table 3: Numerical analysis of solution Eq. (50), (55) and exact solution (56) for example 3 at $\alpha=1$

\begin{tabular}{|c|c|c|c|c|c|c|}
\hline \multirow[t]{2}{*}{$x$} & \multirow[t]{2}{*}{$\mathrm{t}$} & \multirow[t]{2}{*}{ CRDTM } & \multirow[t]{2}{*}{ CFVIM } & \multirow[t]{2}{*}{ Exact Sol } & \multicolumn{2}{|c|}{ Absolute Error } \\
\hline & & & & & CRDTM & CFVIM \\
\hline 0.05 & $\begin{array}{c}0.01 \\
0.001 \\
0.0001\end{array}$ & $\begin{array}{l}1.020201 \\
1.024801 \\
1.025260\end{array}$ & $\begin{array}{l}1.010333 \\
1.024311 \\
1.025390\end{array}$ & $\begin{array}{l}1.018521 \\
1.024630 \\
1.025250\end{array}$ & $\begin{array}{l}1.6832 \times 10^{-3} \\
1.7151 \times 10^{-4} \\
1.6173 \times 10^{-5}\end{array}$ & $\begin{array}{c}8.1882 \times 10^{-3} \\
3.194 \times 10^{-4} \\
1.4253 \times 10^{-4}\end{array}$ \\
\hline 0.09 & $\begin{array}{c}0.01 \\
0.001 \\
0.0001\end{array}$ & $\begin{array}{l}1.040810 \\
1.045510 \\
1.045980\end{array}$ & $\begin{array}{l}1.038328 \\
1.045373 \\
1.045901\end{array}$ & $\begin{array}{l}1.039081 \\
1.045331 \\
1.045961\end{array}$ & $\begin{array}{l}1.7291 \times 10^{-3} \\
1.7940 \times 10^{-4} \\
1.9001 \times 10^{-5}\end{array}$ & $\begin{array}{l}7.5312 \times 10^{-4} \\
4.2641 \times 10^{-5} \\
6.0021 \times 10^{-5}\end{array}$ \\
\hline 0.1 & $\begin{array}{c}0.01 \\
0.001 \\
0.0001\end{array}$ & $\begin{array}{l}1.046030 \\
1.050751 \\
1.051220\end{array}$ & $\begin{array}{l}1.044503 \\
1.050651 \\
1.051210\end{array}$ & $\begin{array}{l}1.044291 \\
1.050571 \\
1.051200\end{array}$ & $\begin{array}{l}1.7390 \times 10^{-3} \\
1.8241 \times 10^{-4} \\
2.0012 \times 10^{-5}\end{array}$ & $\begin{array}{l}2.1201 \times 10^{-4} \\
8.2451 \times 10^{-5} \\
1.0031 \times 10^{-5}\end{array}$ \\
\hline
\end{tabular}

solution of equation (37) for different values of $\alpha$ with fixed values $0 \leq \mathrm{t} \leq 1,0 \leq \mathrm{x} \leq 1$. (b) Depicts that the solution of CFRDTM of equation (42) for different values of $\alpha$ with fixed values $0 \leq \mathrm{t} \leq 1,0 \leq \mathrm{x} \leq 1$. (c) Depicts the comparison of CFVIM and CFRDTM with exact solution for $\alpha=1$ with fixed values $0 \leq \mathrm{t} \leq 1,0 \leq \mathrm{x} \leq 1$. (d) Displays the comparison of CFVIM and CFRDTM and exact solution with $\alpha=0.5$ for different values $\mathrm{x}$ and $\mathrm{t}$. (e) Demonstrates the comparison of CFVIM and CFRDTM with exact solution for different values $\mathrm{x}$ and $\mathrm{t}$ for $\alpha=1$.

Fig 3 (a) shows surface behavior the solution by CFVIM of equation (50) for different values of $\alpha$ with fixed values $0 \leq \mathrm{t} \leq 1,0 \leq \mathrm{x} \leq 1$. (b) Shows physical behavior of solution by CFRDTM of equation (55) for different values of $\alpha$ with fixed values $0 \leq \mathrm{t} \leq 1,0 \leq \mathrm{x} \leq 1$. (c) Displays the comparison of CFVIM and CFRDTM results with exact solution for $\alpha=1$ with fixed values $0 \leq \mathrm{t} \leq 1,0 \leq \mathrm{x} \leq 1$. (d) Illustrate the comparison of CFVIM and CFRDTM and exact solution with $\alpha=0.5$ for different values $\mathrm{x}$ and $\mathrm{t}$. (e) Confirmations the comparison of CFVIM and CFRDTM with exact solution for different values $\mathrm{x}$ and $\mathrm{t}$ for $\alpha=1$.

Table 1, 2 and 3 numerically explore the comparison of obtained solutions with the exact solution. Absolute errors also determine to show the worth of these techniques. 


\section{Conclusion}

In this study, we successfully applied the conformable fractional differential transform method and conformable variational iteration method on the foam drainage equation, time- fractional Gardner equation and timefractional Fornberg-Whitham equation. We showed the existence and uniqueness of the solutions. The solutions obtained by these methods are compared with the exact solution which is presented in graphs. From the results, given graphs, it is clear that for these models CFVIM gives more accurate results as compare to the CFRDTM using only a few iterates. Numerical results indicate that these techniques are very good results when looked carefully at the graphs and are very powerful, simple and compatible for solving the nonlinear FPDEs and allow us to understand the dynamics of nonlinear mathematical model of propagation. In this work Mathematica software is used for computing the results, tables and graphs. From these conclusions, we can say that the CFVIM with fractional derivative is suitable for examining many problems in the fields of science and engineering.

Conflict of interest: The authors declare no conflict of interest regarding the publication of this paper.

\section{References}

[1] Podlubny I. Fractional-order systems and $\mathrm{PI} / \mathrm{sup} / \mathrm{spl}$ lambda//D/sup/spl mu//-controllers. IEEE Trans Auto cont. 1999;44(1):208-214.

[2] Atangana A. Derivative with a new parameter: Theory, methods and applications. Academic Press; 2015.

[3] Zulfiqar A, Ahmad J. Soliton Solutions of Fractional Modified Unstable Schrödinger Equation Using Exp-Function Method. Res Phys; 2020. p. 103476.

[4] Zulfiqar A, Ahmad J. Exact solitary wave solutions of fractional modified Camassa-Holm equation using an efficient method. Alex Eng J. 2020;59(5):3565-74.

[5] Singh P, Sharma D. Comparative study of homotopy perturbation transformation with homotopy perturbation Elzaki transform method for solving nonlinear fractional PDE. Nonlinear Eng. 2019;9(1):60-71.

[6] Grover D, Sharma D, Singh P. Accelerated HPSTM: an efficient semi-analytical technique for the solution of nonlinear PDE's. Nonlinear Eng. 2020;9(1):329-37.

[7] Singh P, Sharma D. Convergence and error analysis of series solution of nonlinear partial differential equation. Nonlinear Eng. 2018;7(4):303-8.

[8] Atangana A, Baleanu D, Alsaedi A. New properties of conformable derivative. Open Math. 2015;13(1):889-98.

[9] Cenesiz Y, Kurt A. The solutions of time and space conformable fractional heat equations with conformable Fourier transform. Acta Univ Sapientiae Matem. 2015;7(2):130-40.

[10] Çenesiz Y, Baleanu D, Kurt A, Tasbozan O. New exact solutions of Burgers' type equations with conformable derivative. Waves Random Complex Media. 2017;27(1):103-16.

[11] Ünal E, Gökdoğan A. Solution of conformable fractional ordinary differential equations via differential transform method. Optik (Stuttg). 2017;128:264-73.

[12] Bayram M, Hatipoğlu VF, Alkan S, Das SE. A solution method for integro-differential equations of conformable fractional derivative. Therm Sci. 2018;22(1 Suppl. 1):7-14.

[13] Mozaffari FS, Hassanabadi H, Sobhani H, Chung WS. Investigation of the Dirac equation by using the conformable fractional derivative. J Korean Phys Soc. 2018;72(9):987-90.

[14] Hosseini K, Bekir A, Ansari R. New exact solutions of the conformable time-fractional Cahn-Allen and Cahn-Hilliard equations using the modified Kudryashov method. Optik (Stuttg). 2017;132:203-9.

[15] Yavuz M. Novel solution methods for initial boundary value problems of fractional order with conformable differentiation. Int J Optimiz Cont Theory App. 2017;8(1):1-7.

[16] Yavuz M, Yaşkıran B. Conformable Derivative Operator in Modelling Neuronal Dynamics. App Appl Math. 2018;13(2):803817.

[17] Yavuz M. Dynamical behaviors of separated homotopy method defined by conformable operator. Konuralp J Math. 2019;7(1):1-6.

[18] He J. A new approach to nonlinear partial differential equations. Commun Nonlinear Sci Numer Simul. 1997;2(4):230-5.

[19] He JH. Variational iteration method-a kind of non-linear analytical technique: some examples. Int J Non-linear Mech. 1999;34(4):699-708

[20] Das N, Singh R, Wazwaz AM, Kumar J. An algorithm based on the variational iteration technique for the Bratu-type and the Lane-Emden problems. J Math Chem. 2016;54(2):527-51.

[21] Acan O, Firat O, Keskin Y. Conformable variational iteration method, conformable fractional reduced differential transform method and conformable homotopy analysis method for nonlinear fractional partial differential equations. Waves Random Complex Media. 2018;30(1):1-19.

[22] Keskin Y, Oturanc G. Reduced differential transform method for partial differential equations. Int J Nonlinear Sci Numer Simul. 2019;10(6):741-50.

[23] Keskin Y, Oturanç G. Application of reduced differential transformation method for solving gas dynamics equation. Int J Contemp Math Sci. 2010;22(22):1091-6.

[24] Srivastava VK, Awasthi MK, Chaurasia RK, Tamsir M. The telegraph equation and its solution by reduced differential transform method. Model Simul Eng. 2013;2013:9.

[25] Acan 0, Al Qurashi MM, Baleanu D. Reduced differential transform method for solving time and space local fractional partial differential equations. J Nonlinear Sci Appl. 2017;10(10):52308.

[26] Gubes M, Keskin Y, Oturanc G. Numerical solution of timedependent Foam Drainage Equation (FDE). Comp Methods Differ Equ. 2015;3(2):111-22.

[27] Islam T, Akbar MA, Azad AK. Traveling wave solutions to some nonlinear fractional partial differential equations through the rational $\left(\mathrm{G}^{\prime} / \mathrm{G}\right)$-expansion method. J Ocean Eng Sci. 2018;3(1):76-81. 
[28] Pandir Y, Duzgun HH. New exact solutions of time fractional Gardner equation by using new version of $\mathrm{F}$-expansion method. Commum Theor Phys. 2017;67(1):9.

[29] Korpinar Z, Inc M, Baleanu D, Bayram M. Theory and application for the time fractional Gardner equation with MittagLeffler kernel. J Taibah Univ Sci. 2019;13(1):813-9.

[30] Ghanbari B, Baleanu D. New solutions of Gardner's equation Using two analytical methods. Front Phys. 2019;7:202.

[31] Iyiola OS, Olayinka OG. Analytical solutions of time-fractional models for homogeneous Gardner equation and nonhomogeneous differential equations. Ain Shams Eng J. 2014;5(3):999-1004.

[32] Ibis B, Bayram M. Analytical approximate solution of time fractional Fornburg-Whithim equation by the fractional iteration method. Alex J Eng. 2014;53(4):911-5.

[33] Singh J, Kumar D, Kumar S. New treatment of fractional Fornberg-Whitham equation via Laplace transform. Ain Shams Eng J. 2013;4(3):557-62.

[34] Zeng DQ, Qin YM. The Laplace-Adomian-Pade technique for the seepage flows with the Riemann-Liouville derivatives. Commun Fract Calc. 2012;3(1):26-9.

[35] Kumar D, Singh J, Baleanu D. A new analysis of the FornbergWhitham equation pertaining to a fractional derivative with Mittag-Leffler-type kernel. Eur Phys J Plus. 2018;133(2):1-10.

[36] Gao X, Lai S, Chen H. The stability of solutions for the Fornberg-Whitham equation space. Bound Value Probl. 2018;2018(1):142.

[37] Alderremy AA, Khan H, Shah R, Aly S, Baleanu D. The Analytical Analysis of Time-Fractional Fornberg-Whitham Equations. Math. 2020;8(6):987.

[38] Mainardi F. Fractional calculus. Fractals and fractional calculus in continuum mechanics. Vienna: Springer; 1997:291-348.

[39] Khan Y, Faraz N, Yildirim A, Wu Q. Fractional variational iteration method for fractional initial-boundary value problems arising in the application of nonlinear science. Comput Math Appl. 2011;62(5):2273-8.

[40] Caputo M. Linear models of dissipation whose $Q$ is almost frequency independent-II. Geophys J Int. 1967;13(5):529-39.

[41] Abdeljawad T. On conformable fractional calculus. J Comput Appl Math. 2015;279:57-66. 